\title{
Quand les instruments de métier deviennent objets de discours
}

Une condition de l'analyse du travail en autoconfrontation croisée?

Turning work tools into objects of discourse: a condition for the analysis of activity using cross self-confrontation?

\section{Laure Kloetzer et Muriel Henry}

\section{OpenEdition}

\section{Journals}

Édition électronique

URL : http://journals.openedition.org/activites/2406

DOI : $10.4000 /$ activites. 2406

ISSN : 1765-2723

\section{Éditeur}

ARPACT - Association Recherches et Pratiques sur les ACTivités

\section{Référence électronique}

Laure Kloetzer et Muriel Henry, «Quand les instruments de métier deviennent objets de discours », Activités [En ligne], 7-2 l octobre 2010, mis en ligne le 15 octobre 2010, consulté le 01 mai 2019. URL http://journals.openedition.org/activites/2406 ; DOI : 10.4000/activites.2406

\section{(c) (i) () $\Theta$}

Activités est mis à disposition selon les termes de la licence Creative Commons Attribution - Pas d'Utilisation Commerciale - Pas de Modification 4.0 International. 


\title{
Quand les instruments de métier deviennent objets de discours :

\author{
une condition de l'analyse du travail
} en autoconfrontation croisée?
}

\author{
Laure Kloetzer et Muriel Henry \\ Équipe clinique de l'activité, Centre de Recherche Travail et Développement (EA 4132) \\ CNAM, 1 rue Gay Lussac - 75005 Paris \\ laure.kloetzer@gmail.com ; Muriel.Henry@u-bourgogne.fr
}

\begin{abstract}
Turning work tools into objects of discourse: a condition for the analysis of activity using cross self-confrontation? A growing number of work analysis methodologies demand a developmental approach, i.e. they aim to change workers and/or work situations. Some assume that this change can best be achieved by closely involving people in the analysis of their own work activity (e.g. Clot, 2008; Engeström, 1987, 2000; Béguin \& Cerf, 2004; Pastré, 2005). However, research experience shows that examples of such change remain uncommon, even in frameworks carefully designed to support the subjects' development of their activity, not to mention the development of practical activity, which is generally very difficult to demonstrate within the space and time restrictions of a research project. In this paper, we analyse a few such examples drawn from two projects carried out in Clinic of Activity using the technique of cross self-confrontation (Clot, 1999, 2005, 2008; Clot et al., 2001). The first was conducted in conjunction with experts in Accreditation for Work Experience (or VAE, as it is known in French), the second with Roman Catholic priests. We compare the two to acquire a better understanding of the conditions required for development of the analysis. We will focus on one of the mechanisms that recurs often in our material: the construction of a "space for reflection" (Perret-Clermont, 2001) where new ways of thinking are created.
\end{abstract}

\section{KEYWORDS}

Work analysis, activity, dialogical frameworks, cross self-confrontation, development, functional migration.

Dans le cadre des travaux qui associent étroitement les professionnels à l'analyse de leur activité dans une visée de transformation de cette dernière, la mobilisation du langage constitue désormais une caractéristique essentielle dans l'espace des débriefings, des retours d'expérience, du "Change Lab » (Engeström, Virkkunen, Helle, Pihlaja, \& Poikela, 1996), ou des autoconfrontations, par exemple. Les recherches de terrain s'accompagnent de la production de nombreux discours sur le travail. Ces discours sont d'origine variée, en provenance des professionnels eux-mêmes, de leurs chefs, de leurs collègues, ou encore des chercheurs mandatés pour conduire l'analyse. Ils ont également des destinataires variables : les chercheurs eux-mêmes qui posent les questions, les commanditaires de la recherche, les collègues présents dans la situation d'interaction, ou au-delà. Dans la maille de ces discours complexes se mêlent en outre des caractéristiques très diverses de l'activité de travail : ses conditions préalables, ses temporalités, ses difficultés, ses instruments (Bronckart \& Bulea, 2006 ; Bulea, 2007). Si ces discours sont très variés dans leur nature, leurs rapports avec l'activité qu'ils commentent le sont tout autant, ce qui leur confère un statut parfois ambigu (Filliettaz \& Bronckart, 
2005). Nous nous intéressons ici spécifiquement à une méthode développementale usant du langage, celle des autoconfrontations croisées. Nous cherchons à comprendre les conditions dans lesquelles l'activité langagière de sujets impliqués dans le cadre dialogique des autoconfrontations croisées leur permet de mettre en place une analyse de leur activité de travail ouvrant à des développements potentiels de cette dernière ${ }^{1}$.

\section{1.- L'autoconfrontation croisée comme dispositif d'analyse du travail}

L'autoconfrontation croisée vise à initier une démarche réflexive orientée vers le développement des travailleurs et des situations de travail au sein d'un collectif professionnel. Elle s'appuie sur le langage, et sur l'image, à travers la réalisation de films de l'activité. Méthodologiquement, il s'agit de considérer que l'expérience vécue n'est jamais accessible directement, comme Vygotski le montre, en critiquant les postures objectivistes et subjectivistes en psychologie (Vygotski, 1999). L'expérience ne se laisse pas saisir sur le vif pour la raison même que l'action qu'on exerce sur elle, pour y accéder, l'affecte en retour. Ainsi les effets de l'observation de l'activité sur cette dernière sont couramment repérés dans les interventions, mais souvent ignorés dans les analyses ${ }^{2}$. De même la réalisation langagière, avec sa spécificité fonctionnelle, affecte l'expérience dont il est question. Notamment, elle la réalise à chaque fois différemment selon l'interlocuteur.

\section{1.- La variation des destinataires au cour du dispositif}

Le dispositif d'autoconfrontation croisée (Clot, 1999, 2005, 2008 ; Clot, Faïta, Fernandez, \& Scheller, 2001) s'appuie sur les effets induits par le changement de destinataire pour favoriser la réflexion individuelle et collective sur l'activité de travail. Il organise pour cela une reprise dans différents contextes langagiers de l'analyse du travail. Il se déploie en trois temps :

- Premièrement, observation in situ de l'activité par le chercheur, construction d'un collectif de professionnels volontaires associé à l'analyse et choix des séquences clefs d'activité à analyser ;

- Deuxièmement, construction et commentaires des films d'activité ;

- Troisièmement, retour dans le collectif associé à l'analyse.

Les autoconfrontations croisées sont surtout connues pour la succession des deux phases de commentaires différemment adressés qu'elles organisent au deuxième temps de la méthode :

- Un premier entretien, appelé autoconfrontation simple, permet au professionnel d'observer et de commenter le film de son activité avec le chercheur. Le regard et les questions du chercheur permettent au professionnel de commencer à déplacer son propre regard sur ce qu'il fait à travers ce qu'il se voit faire à l'écran ;

- Un deuxième entretien, appelé autoconfrontation croisée, invite deux professionnels et le chercheur à dialoguer sur leurs diverses manières de faire à partir de l'observation fine de leurs deux films d'activité. Ce dialogue est croisé, dans la mesure où nous regardons successivement chacun des films d'activité, en proposant systématiquement au professionnel dont l'activité n'est pas présente sur le film de commenter le film de son collègue en lui faisant part de ses interrogations et de ses impressions. Le regard posé sur l'activité n'est plus seulement celui du chercheur mais du pair, et cette double adresse permet au professionnel de continuer à déplacer son propre regard. Dans la comparaison fine de manières de faire différentes pour traiter les

1. La question des développements induits de l'activité pratique ne sera donc pas traitée dans cet article.

2. Wallon l'a bien montré : Alors, «l'attention que le sujet sent fixée sur lui semble, par une sorte de contagion très élémentaire, l'obliger lui-même à s'observer. S'il est en train d'agir, l'objet de son action et l'action elle-même sont brusquement supplantés par l'intuition purement subjective qu'il prend de son propre personnage. C'est comme une inquiétude, une obsession de l'attitude à adopter. C'est un besoin de s'adapter à la présence d'autrui, qui se superpose à l'acte en cours d'exécution [...] » (Wallon, 1983, p. 287). 
mêmes objets et choix professionnels, dans des situations voisines, se révèlent des traits et problèmes fondamentaux du métier et s'élargissent potentiellement les ressources pour y faire face.

Dans le dispositif méthodologique, l'adresse du discours varie, le cadre mobilisant explicitement des interlocuteurs différents : d'abord en autoconfrontation simple, le chercheur qui tente de comprendre ; puis en autoconfrontation croisée, le pair du même métier qui ne s'en laisse pas conter sur celui-ci et avec qui peuvent s'engager des controverses ; enfin l'ensemble du groupe de volontaires, lors des discussions collectives sur les données collectées ${ }^{3}$. C'est dans la mesure où ces discours sont d'abord adressés à d'autres, agissant sur d'autres (par exemple en tentant de les convaincre) que les discours peuvent constituer un moyen d'action sur soi, moyen psychique de réalisation de la pensée (Vygotski, 1997) et d'organisation de l'action (Vygotski, 1978).

\section{2.- Analyse du travail et activité langagière}

Comme dans d'autres dispositifs, le langage n'est là jamais un but de l'activité - on ne parle pas pour parler. Dans le cadre de l'autoconfrontation croisée, l'activité langagière est au service d'une activité d'analyse, de pensée nouvelle sur le travail, activité qui en retour alimente celle de l'échange avec le chercheur et les pairs (Kostulski, 2005). Si à la suite de Vygotski, on peut avancer que le langage réalise à chaque fois différemment la pensée ${ }^{4}$, la richesse de ce rapport quand il porte sur l'activité pratique de travail incite à développer les conditions et les processus qui sont engagés. D'autant que les constats empiriques n'indiquent aucune automaticité : les acteurs peuvent mobiliser dans les échanges un «prêt à penser» ou un «déjà dit». Si les méthodes développementales ont en commun de s'engager de plain pied dans la mobilisation du langage comme moyen de réaliser leur dessein, le statut et les fonctions de celui-ci ne sont généralement pas au centre des travaux.

A la suite de K. Kostulski, nous proposons de voir l'autoconfrontation comme « une activité conjointe, médiatisée par un instrument symbolique (Vygotski, 1930/1985) nécessaire à la réalisation de l'activité » (Kostulski \& Clot, 2007, p. 75). Ce qui donne au langage simultanément une triple nature : une activité humaine, par exemple converser, un instrument psychologique de l'activité et un résultat de leur mise en œuvre (Kostulski \& Clot, 2007).

Dans ce cadre, nous soutenons qu'il existe des régularités dans le développement des activités d'analyse au moyen du langage, liées à la nature de celui-ci et à ses mouvements. Des travaux antérieurs (Clot, 2004, 2005, 2008 ; Kostulski \& Clot, 2007) ont dégagé l'importance des migrations fonctionnelles dans ce développement. Les migrations fonctionnelles sont les mouvements qui conduisent les différents éléments engagés dans l'activité à occuper plusieurs fonctions successivement ou conjointement dans l'activité du sujet. Par exemple l'objet - ce sur quoi porte l'activité - peut devenir instrument - moyen de celle-ci (Vygotski, 1978). C'est alors le « caractère nomade » du langage, son passage d'un statut à l'autre qui peut constituer la source des transformations nécessaires à la dynamique du processus conversationnel. Nous montrerons ici un mouvement ou plutôt une succession de mouvements de ce type, qui semble constituer une condition des développements de l'activité d'analyse du travail en autoconfrontation croisée.

\section{2.- Un mouvement de double migration mis en évidence dans deux séquences empiriques}

Les séquences analysées sont extraites d'interventions très différentes dans leurs publics et leurs

3. Nous ne présumons pas des autres interlocuteurs « invisibles », mais présents dans l'histoire du professionnel ou de son milieu de travail, auxquels le professionnel peut se trouver à s'adresser à un moment ou à un autre du dispositif dans son processus de réflexion.

4. Du coup, chacun en vient à « percevoir les choses autrement, et en même temps à acquérir d'autres possibilités d'action par rapport à elles. Comme devant un échiquier, je vois autrement, je joue autrement » (Vygotski, 1997, p. 317). 
objets. Nous présenterons d'abord une séquence extraite d'une autoconfrontation simple (par la suite ACS) conduite lors d'une intervention réalisée avec des professionnels de la Validation des Acquis de l'Expérience (VAE), puis une séquence extraite d'une autoconfrontation croisée (par la suite ACC) conduite avec des prêtres catholiques. Dans chacune de ces séquences, nous analyserons deux occurrences d'un mouvement de double migration. Ceci nous permettra de les comparer d'abord l'une l'autre au sein de la séquence (comparaison intra séquence) puis les unes avec les autres entre les deux séquences présentées (comparaison inter séquences).

Les données empiriques présentées comprennent donc :

- Une première séquence $\mathrm{S} 1$, issue d'une $\mathrm{ACS}$, dite «alors je ne sais pas si c'est guider...» où la double migration se produit autour de la reprise des termes « guider » puis « reconnaissance »;

- Une deuxième séquence S2, issue d'une ACC et dite séquence «Tu n'adoreras que le Seigneur ton Dieu », où la double migration concerne les notions de « traduction liturgique » et de « chemin ».

\section{1.- Analyse de la séquence S1 (extrait d'Autoconfrontation simple) dite séquence « alors je ne sais pas si c'est guider »}

La recherche est développée à partir d'une intervention commanditée par un Ministère qui met en place un nouveau dispositif de certification, la Validation des Acquis de l'Expérience ${ }^{5}$. Inédit dans l'histoire de l'éducation en France, celui-ci permet désormais d'obtenir un diplôme à partir de la seule expérience professionnelle, sans plus de lien avec un quelconque dispositif de formation. Dans ce cadre de travail nouveau, il s'agit concrètement pour les professionnels, déjà formateurs ou évaluateurs, de délivrer tout ou partie du diplôme sollicité à partir d'un dossier constitué des expériences professionnelles que les candidats formalisent et analysent. Cette activité pose de nombreuses questions (par exemple, comment s'assurer de la réalité des compétences ?) et plus encore de problèmes, sensibles mais peu dicibles. Dans l'ensemble du processus de validation des acquis de l'expérience qui conduit un membre de jury de la lecture du dossier à la décision de certification, le collectif a choisi en particulier de travailler sur un moment qui pose beaucoup de questions à tous ${ }^{6}$, l'entretien avec le candidat, qui fait l'objet de la séquence S1 présentée ci-dessous. L'activité d'entretien entre dans un cadre de prescription très détaillé qui en précise la durée (pas plus d'une demi-heure), la nature (ce n'est ni un oral de rattrapage ni une soutenance), et les modalités.

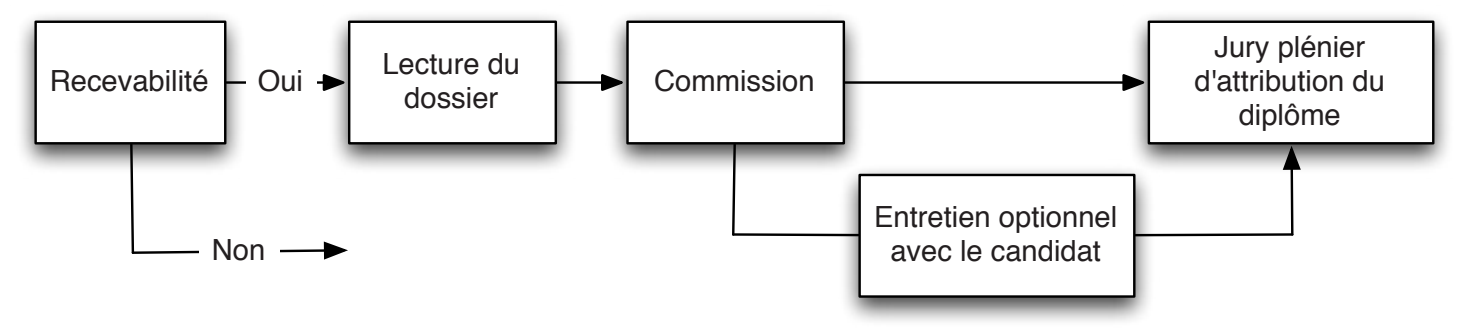

Figure $1:$ Le processus de validation des acquis de l'expérience Figure 1: The procedures involved in Accreditation for Work Experience (French
VAE).

Le texte que nous analysons est issu du commentaire du professionnel (JF, un formateur très expérimenté qui fonde sa pratique sur les valeurs de l'éducation populaire ${ }^{7}$ ) en autoconfrontation simple sur l'activité qui le conduit avec une

5. Décret sur la validation des acquis de l'expérience, 2002

6. Par exemple, comment commencer l'entretien, quelles questions poser...

7. «Former des citoyens actifs et responsables par une pédagogie adaptée favorisant la créativité est la finalité de l'éducation populaire. L'éducation populaire est un moyen et une méthode de l'éducation à la citoyenneté. Elle reconnaît et associe une dimension humaniste de développement de l'individu (selon son parcours de vie, son environnement) et 
collègue à entendre une candidate à la VAE. Le commentaire porte sur les toutes premières minutes du film de l'entretien. Nous présentons ici les premiers échanges entre le chercheur et JF, qui s'engagent après que le premier a rappelé la nature de la tâche ${ }^{8}$ et le second testé le matériel de visionnage. Les films de l'activité de travail et ceux des dialogues engagés en autoconfrontation constituent la source des traces ici retranscrites.

\subsection{1.- La séquence $S 1$}

JF commence à commenter le film de son activité d'entretien avec la candidate en soulignant l'importance de mettre les candidats à l'aise dans cette situation d'évaluation de leurs acquis professionnels. Le film est alors arrêté sur l'image des deux membres de jury - dont lui-même - qui attendent que la candidate s'installe en lui « laissant le temps » de le faire.

JF 6 oh ça...ça se passe ...généralement comme ça hein je veux dire ...bon...si, c'est vrai on les... on les... on leur dit de s'asseoir... et puis de se mettre à l'aise... on les laisse un petit peu s'installer c'est vrai on les... on prend le temps là c'est vrai... on commence effectivement le décomptage de l'entretien d'une demi-heure que...lorsque ces petites choses là ont été réglées donc ... pour nous ça fait partie aussi de....je veux dire de la des conditions dans lequel... on rassure le candidat sur ce qui va... quoi sur les conditions dans lesquelles ça se déroule... non non on cherche plutôt à le mettre à l'aise moi en tout cas c'est ...c'est ce que j'essaie de faire hein... de mettre à l'aise le candidat donc... ben... en lui... en essayant quand même d'être attentif par le regard et d'être... et de montrer qu'on est quand même déjà à l'écoute de ce qu'ils peuvent dire... essayer d'être le plus à l'écoute possible. mais uniquement par... plus par l'attitude que par la parole

Ch $\quad 7 \quad$ c'est important que les candidats soient à l'aise pour ...

JF 8 oui oui

Ch 9 pour la suite?

JF 10 je pense oui c'est important... en tout cas dans toutes les conditions que ce soit VAE ou une autre condition d'examen des oraux, on essaie de mettre ... le candidat à l'aise. Et aussi chose qu'on fait toujours c'est se présenter, dire... à quel titre ... on parle donc...donc....quand on est membre du jury c'est pour les candidats c'est important de savoir... aussi...quel est le statut quoi la position dans le jury... si c'est ....je dirais un représentant des syndicats employeurs des syndicats employés là c'était une une... formatrice mais ça aurait pu aussi... Les candidats je pense quelque part derrière ça, ils ont... ça peut aussi les guider (sourire) ça les aide et puis c'est pas c'est pas un entretien anonyme (inaudible)

Ch 11 vous dites que ça peut les guider?

JF 12 alors je sais pas si c'est guider mais enfin c'est nous c'est. Bon . je sais pas j'ai pas trop analysé. En tout cas, on a l'habitude de le faire (rire) c'est pour ça et ...on se pose rarement la question de savoir .... Non c'est d'où t'on d'où on parle je crois je veux dire on est aussi ....'est un acte qu'on fait en pédagogie aussi on s'exprime toujours ... de ... de quelque...enfin on dit toujours de ... qui on est quand on ... donc on n'est pas dans une attitude vraiment pédagogique puisque là on est dans la certification on n'est pas en formation mais... ou dans l'évaluation mais... je pense ça a une importance pour les personnes de savoir ...ben qui on est aussi ça fait partie des, de ce qu'on peut enseigner aussi (rire) le savoir être a tellement d'importance dans nos... dans nos métiers, et dans les métiers qu'ils... que font aussi les candidats les animateurs que c'est important de savoir qui ils ont en face

JF poursuit ensuite son commentaire sur la séquence suivante du film : les deux membres du jury se présentent, indiquent de quelle manière l'entretien va se dérouler. Dans la situation, JF précise

une dimension politique d'émancipation (place de l'individu dans la société) ».

8. Le professionnel est généralement invité à commenter ce qu'il fait, en arrêtant le déroulement du film par exemple s'il est surpris ou souhaite expliciter quelque chose. 
que le dossier qu'a transmis la candidate est « très riche » et qu'on ne reviendra donc pas sur tout. Il propose de poser quelques questions en rappelant que le dossier a été lu par une commission de plusieurs personnes et que les deux membres de jurys sont là « délégués » par cette commission pour poser les questions.

Ch 33 vous reprécisez que vous n'êtes pas les deux seules personnes impliquées c'est ... important?

JF $\quad 34$ oui pour le candidat je pense que.... Ca c'est ... c'est c'est... c'est... pour ... lui montrer qu'il y a eu des regards croisés quoi je veux dire sur son... sur son dossier qu'il a été lu par... attentivement par des personnes différentes et que... bon c'est pas... c'est c'est ... que ça fait l'objet de discussions bon on le dit pas mais j'espère que... c'est implicite pour tout le monde mais enfin... on espère j' espère que c'est implicite ...implicite aussi pour la candidate que son dossier a été.... Rappeler quoi je veux dire l'intérêt qu'on a pu porter, voilà je veux dire je crois que c'est important ...quand on voit ben l'épaisseur... bon le travail qu'elle a pu réaliser, sur ce dossier là moi je crois que c'est c'est déjà bon c'est une forme de reconnaissance quoi je veux dire en disant ben le dossier n'a pas été lu par une personne sur un coin de table et puis voilà on a préparé un questionnement... rapidement non. Y'a.... une commission et cette commission en plus... a soumis ça... a soumis son avis à une autre... parce qu'il y a deux commissions en parallèle ... parce qu'on avait beaucoup de... dossiers donc... et donc... pour aussi.... des questions d'équité entre tous les candidats. Les deux commissions lorsqu'elles ont fini leur travail de lecture et déjà $\mathrm{d}$... avoir élaboré.... des avis// /.../

ch 35 et le dire à la candidate vous disiez c'est une forme de reconnaissance par rapport à elle?

JF 36 ben oui du... je veux dire du travail qu'elle a (amorce de rire) pu accomp accomplir quoi alors reconnaissance... je sais pas si c'est le mot mais enfin en tout cas ... dire que... son dossier a été... lu avec intérêt (rire) et que...on y a porté cas et que... c'est c'est important qu'il y ait plusieurs lecteurs quoi . Je sais pas. C'est aussi pour... comment je vais dire... éviter... oui que... que... la subjectivité quoi je veux pas dire qu'on est objectif (rire) mais on essaie d'avoir d'aller vers le plus d'objectivité possible donc... en disant que le... le nombre de lecteurs est plus important c'est..., je pense... pour... pour le candidat ça doit le ...le... comment je vais dire...ça doit être plus im c'est important pour qu'il comprenne que c'est plus objectif... c'est pas sûr hein ... (rire) là j’ai aucun moyen de vérifier si elle a bien compris ça mais enfin bon c'est... c'est dit dans cet inter...dans ce cadre là

\subsection{2.- Analyse détaillée des mouvements de double migration}

\section{Premier mouvement : autour de « guider»}

Durant les premières minutes de son commentaire, JF explicite ce qui n'est pas visible à l'écran, à savoir les raisons qui font que sa collègue comme lui-même attendent sans rien dire ni faire tandis que la candidate s'installe. Durant cette phase de l'autoconfrontation, les professionnels découvrent que malgré la recherche d'un commentaire au plus près des images et leurs détails, les points les plus importants ne se voient pas. Le résultat en est souvent l'énonciation de la manière dont on fait en général dans cette situation. C'est ce que JF réalise conversationnellement. Il avance que « les jurys aident toujours les candidats » et conclut que cela peut les guider. A ce point, le dialogue peut se poursuivre sur la même modalité qui justifie par le générique ce que l'on voit de singulier à l'image. Le chercheur a alors souvent à intervenir, de plusieurs manières possibles. Ici, il reprend le mot prononcé par JF. Ce sont précisément ces trois tours de parole (10 à 12) et leurs suites que nous analysons ici en détail. On peut en caractériser le mouvement pas à pas.

- Une signification, qu'on pourrait nommer « l'aide aux personnes », et dont on peut soutenir qu'elle appartient au genre de l'éducation populaire, se développe au travers de plusieurs mots. «L'aide aux personnes » constitue dans l'activité de travail des formateurs de cette tradition un 
« concept organisateur» (Pastré, 2005) et à ce titre constitue un moyen du travail qui oriente les actions. Dans l'activité d'autoconfrontation, il demeure un instrument mais cette fois au service d'une activité de justification ou d'argumentation auprès du chercheur de ce qui est vu à l'écran.

- Un mot parmi ceux utilisés, « guider» (qu'accompagne un sourire de JF) est repris par le chercheur, qui l'extrait ainsi du flux du dialogue engagé. Ce n'est plus vers l'image que le commentaire de JF est tourné mais vers ce mot : celui-ci était un moyen de justifier auprès d'autrui, il devient objet même de l'activité engagée dans l'autoconfrontation.

- Alors le flux discursif s'interrompt, le mot s'interroge (je ne sais pas si c'est le mot) pour laisser place à des hésitations, des silences. Cette difficulté à dire signe à rebours la constitution particulière de l'objet de l'activité en « objet de discours » si on définit celui-ci comme « le lieu où se croisent, se rencontrent, se séparent des points de vue différents » (Bakhtine, 1984, p. 301). Le sens du terme se délie de ses références habituelles et s'interroge.

On peut représenter ainsi ce mouvement :

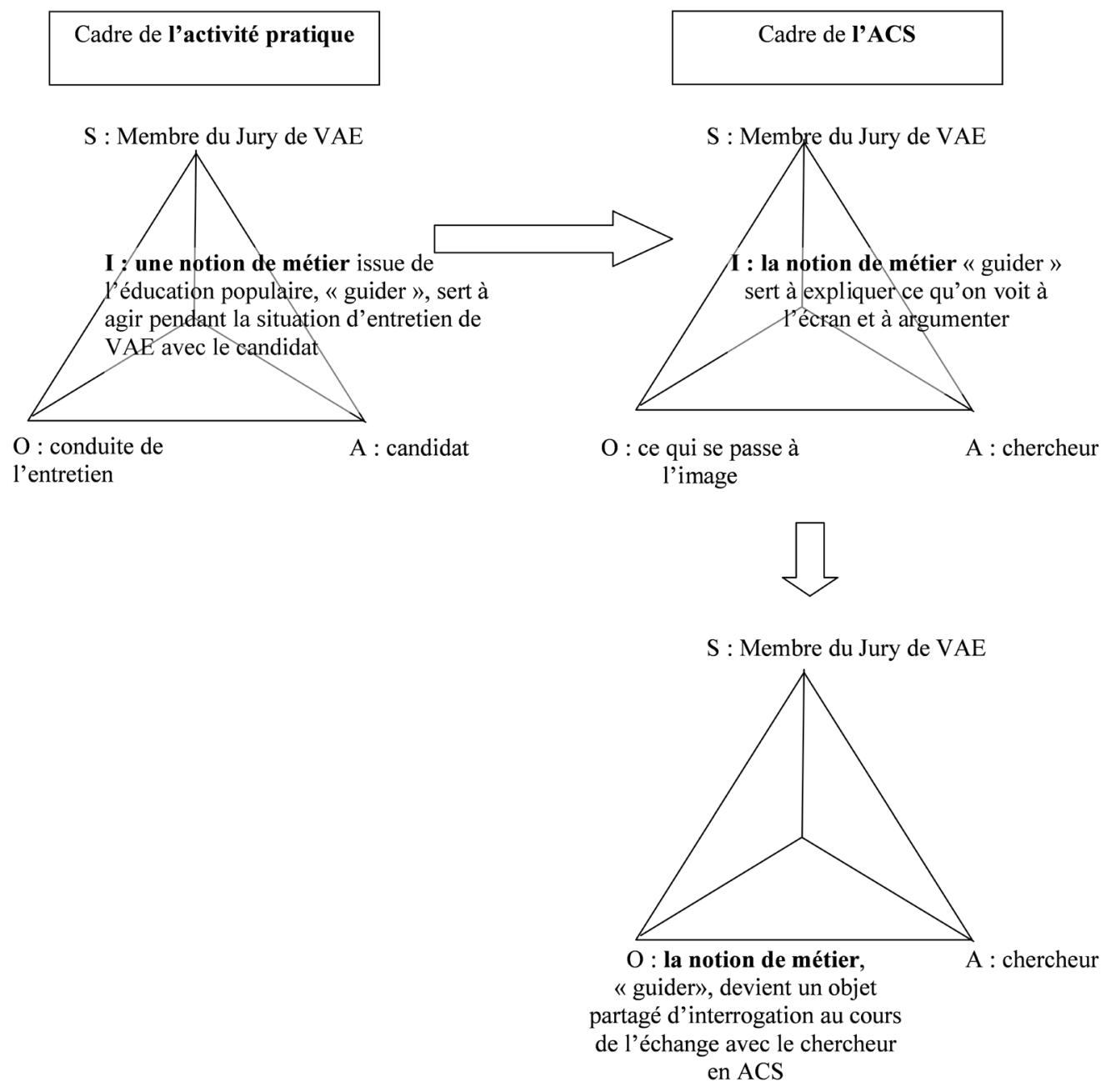

Figure 2 : De l'instrument de métier à l'objet du discours : le cas de « guider »

Figure 2: From work tool to object of discourse : the case of "guiding»

Dans ce schéma comme dans tous les suivants, les pôles du triangle de l'activité du sujet désignent respectivement : S, le sujet ; O, l'objet de l'activité ; A, autrui, partenaire dans l'activité ou destinataire de cette dernière. Et la lettre I représente les instruments mobilisés pour réaliser l'activité. 


\section{Deuxième mouvement : autour de « reconnaissance »}

Un mouvement similaire, au moins dans ses débuts, se répète quelques minutes plus tard, autour du mot « reconnaissance ». Celui-ci actualise une signification qui fait partie d'une même famille, celle de l'éducation populaire, et que l'on pourrait nommer «la considération des personnes ». Le mouvement se produit également sur trois tours de parole (34 à 36). Schématiquement, ceci donne :

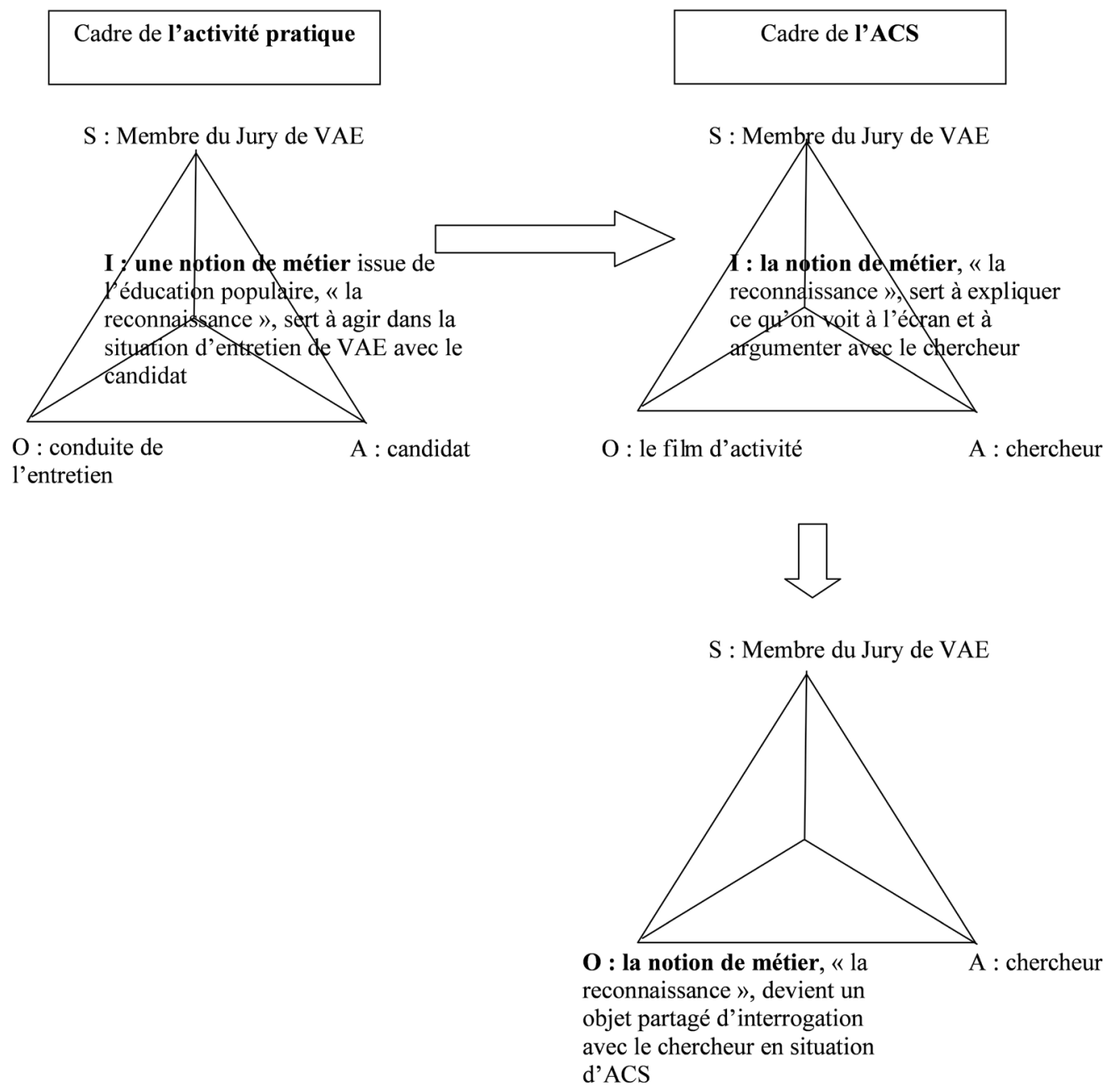

Figure 3 : De l'instrument de métier à l'objet du discours : le cas de « reconnaissance»

Figure 3: From work tool to object of discourse : the case of «recognition»

On peut confirmer une homologie entre les mouvements observés de 10 à 12 et de 34 à 36 : dans les deux cas, un instrument de métier utilisé pour conduire l'activité pratique d'entretien d'abord mobilisé dans l'ACS comme moyen d'argumenter auprès du chercheur à propos de ce qui est fait et vu à l'écran, devient à la suite de la reprise de sa réalisation langagière un objet d'interrogation.

En premier effet, dans les deux cas, le mot se dénaturalise (je ne sais pas si c'est le mot) et le discours change de régime avec de nombreuses hésitations et des silences. Dans un second temps, c'est une autre épaisseur de l'activité qui se révèle aux yeux du chercheur comme à ceux du professionnel. La première situation semble faire émerger aux yeux de JF de nouvelles « réalités » de son activité de membre de jury de VAE (par exemple, il réalise la différence entre ce qu'il fait habituellement comme pédagogue et la nouvelle situation professionnelle dans laquelle il se trouve « on n'est pas en formation » (12). La seconde situation conduit à adjoindre à l'instrument habituel de métier « aide 
et attention aux personnes » une autre dimension propre à la situation d'évaluation, «l'objectivité ». Les suites de l'autoconfrontation permettent de penser qu'il s'agit là de l'expression d'un conflit de métier central - invisible jusque là - dans cette activité de jury de VAE, entre attention portée aux personnes et exigences d'une situation certificative.

Ces observations, même répétées, pourraient être le fruit d'une caractéristique individuelle de JF, dont les formes de commentaire seraient toujours identiques. Toutefois, un autre travail en autoconfrontation croisée, réalisé à partir d'autres activités (Kloetzer, 2008), conduit à repérer un processus analogue.

\section{2.- Présentation et analyse de la séquence $S 2$ (extrait d'autoconfrontation croisée) dite séquence « Tu n'adoreras que le Seigneur ton Dieu »}

La recherche est développée à partir d'une intervention originale, conduite avec un collectif de huit prêtres du diocèse de Paris intéressés par une co-analyse approfondie de leur façon de faire une homélie. L'homélie désigne le moment où, dans le cadre de la messe, le prêtre prend publiquement la parole pour commenter pour l'assemblée les textes bibliques qui viennent d'être lus. L'homélie se découpe en fait en deux temps bien distincts :

- Celui de sa préparation, qui se déroule en général pour les prêtres diocésains sur une semaine, du lundi au samedi ${ }^{9}$, et qui comprend une lecture des textes bibliques du dimanche à venir suivie d'une période de maturation et de préparation de l'homélie (c'est-à-dire d'écriture d'un canevas pour le discours, qu'il s'agisse seulement des points clefs du plan, de notes parcellaires ou de l'intégralité du texte de l'homélie);

- Et celui de sa délivrance pendant la messe où le prêtre prend librement la parole pendant une quinzaine de minutes.

L'homélie est délicate puisqu'elle impose au prêtre de parvenir à faire le lien entre des textes bibliques souvent éloignés en apparence des réalités sociales et l'expérience ordinaire des paroissiens. La méthode des autoconfrontations croisées a été retenue comme un moyen d'objectiver et de discuter les différences de pratique.

Nous nous intéressons ici à une séquence brève extraite de l'entretien en autoconfrontation croisée entre deux prêtres, ci dessous désignés par M et A. L'entretien en autoconfrontation croisée fait suite aux deux autoconfrontations simples de $\mathrm{M}$ et de A réalisées séparément avec le chercheur. Chaque prêtre y commentait pour le chercheur les images de son homélie. En autoconfrontation croisée, les deux prêtres sont simultanément en position de visionner et commenter le film de l'homélie de leur confrère, toujours avec la participation du chercheur ${ }^{10}$. Dans la séquence retenue, A commente les images de l'homélie de M. Ce commentaire prend sa source dans l'intérêt qu'A éprouve pour le travail de $\mathrm{M}$ qu'il trouve très réussi. Il dira en conclusion de cette séquence de l'homélie de son confrère que c'est « du grand art » (en 34 dans la séquence S2). Ce que A admire dans l'homélie de M c'est particulièrement le «croisement » (en 1) qu'elle organise entre l'univers social et le texte biblique. Tout l'objet du questionnement initial d'A est de comprendre comment $\mathrm{M}$ a construit son homélie afin de réussir ce croisement, et plus précisément, comment l'idée même de ce croisement lui est venue. Il le questionne ainsi sur sa préparation de l'homélie qui n'a pas été filmée.

C'est dans ce contexte que $\mathrm{M}$ en vient à parler de sa lecture des textes, et notamment de son usage

9. En effet, les prêtres diocésains prêchent la plupart du temps chaque dimanche. D'un dimanche à l'autre, les textes bibliques lus à la messe changent et donc, le prêtre doit produire une nouvelle homélie qui leur fasse écho. Le temps de la préparation (six jours) peut même être écourté si le prêtre s'y prend seulement en milieu ou en fin de semaine.

10. Le professionnel dont les images n'apparaissent pas à l'écran reçoit des instructions précises pour commenter le film : celles d'arrêter le déroulement du film pour poser des questions à son confrère si quelque chose dans les images de l'activité l'étonne, l'interpelle, lui paraît étrange ou qu'il a besoin d'éclaircissements. 
de la version grecque (en 4). Depuis le concile Vatican II, les textes bibliques sont lus en français, et donc traduits. Toutefois, M privilégie une lecture contrastée de cette version liturgique française et de la version originale de l'Evangile (en grec ancien). Pour expliquer à son confrère ce que cette dernière lui apporte, $\mathrm{M}$ en vient à évoquer un exemple récent qu'il a en tête (en 10). Sur quoi $\mathrm{A}$ s'intéresse à ce nouvel élément en tant que tel et demande des précisions (en 11) ce qui amène $M$ à développer l'exemple d'interprétation du texte biblique qui n'était à l'origine qu'une illustration de son propos sur la préparation de son homélie.

Nous nous intéressons à deux moments distincts de ce bref échange :

— Tout d'abord, l'intervention (en 11) de A « Dis moi dis moi ça m'intéresse », en transformant sa position dans le dialogue, provoque l'ouverture d'une « parenthèse » conversationnelle dans laquelle $\mathrm{M}$ tente de rendre compte de ce qu'il fait non plus en général mais en expliquant un exemple spécifique.

- Par ailleurs, la reprise (en 26) par le chercheur d'une expression employée par M («le chemin ») et du geste qui lui est associé et qui reproduit en quelque sorte ce zigzag dans l'espace, permet de faire entrer, dans l'espace de l'analyse, cette notion de métier qu'est la question du plan, de l'itinéraire que dessine l'homélie.

\subsection{1.- La séquence $\mathrm{S} 2$}

\begin{tabular}{|c|c|c|}
\hline A & 1 & Comment tu as préparé ? Comment t'est venu ce croisement comment (...)? \\
\hline M & 2 & En fait quand je prépare une homélie bon je commence par lire les lectures \\
\hline A & 3 & Oui c'est pas mal (rires) c'est une bonne idée \\
\hline M & 4 & $\begin{array}{l}\text { Et alors je lis toujours l'Evangile en grec. Il faut vraiment que je sois archi charrette pour ne pas } \\
\text { le faire }\end{array}$ \\
\hline A & 5 & Tu le lis quand? \\
\hline M & 6 & J'essaye le lundi j'essaye de le faire le lundi \\
\hline A & 7 & Mmm \\
\hline M & 8 & Et après ça même si je fais absolument autre chose \\
\hline A & 9 & Ca travaille \\
\hline M & 10 & $\begin{array}{l}\text { Voilà. Donc euh il y a les textes et il y a l'Evangile en grec et et éventuellement des notes } \\
\text { exégétiques quand même de temps en temps je vois des trucs par exemple certains mots clefs } \\
\text { dimanche prochain il y a une histoire d'adoration si tu m'adores etc. et en fait euh en fait } \\
\text { la traduction liturgique recouvre... Bon bref }\end{array}$ \\
\hline A & 11 & Dis-moi dis-moi ça m'intéresse \\
\hline M & 12 & $\begin{array}{l}\text { Le démon dit voilà dans la traduction liturgique « si tu te prosternes devant moi pour m'adorer } \\
\text { et ensuite Jésus lui dit «tu n'adoreras que le Seigneur ton Dieu c'est lui que tu adoreras tu ne } \\
\text { te prosterneras que devant le Seigneur ton Dieu et c'est lui que tu adoreras ». Bon. Et en réalité } \\
\text { ''est (pesson proskulein) c'est-à-dire tombant le démon il dit tombant tu te prosternes enfin tu } \\
\text { m'adores tombant tu m'adores et Jésus lui répond «tu n'adoreras que le Seigneur ton Dieu et à } \\
\text { lui seul tu rendras un culte» }\end{array}$ \\
\hline
\end{tabular}

A 13 Ouais

M 14 Donc en fait il y a un déplacement là où ça s'arrête dans la proposition du démon. Tombant tu m'adoreras. Ca s'arrête. Là dans la réponse de Jésus il y a un mouvement : tu adores et tu rendras un culte qui ouvre à à toutes sortes de choses qui ouvre au culte de la vie etc. Donc c'est beaucoup plus intéressant que de dire adore-moi ben non tu n'adoreras que Dieu. D'ailleurs justement souvent c'est à partir d'un point d'un point clef comme ça qui me paraît très savoureux en soi que je me dis il faut que je donne à entendre ça. Alors ensuite ça c'est la première phase voilà l'Evangile j'ai entendu ça il faut que je donne à entendre ça. Et alors après ça justement il faut que je trouve quelque chose qui est de l'ordre de l'expérience commune qui peut être amené par une citation littéraire par un par un fait de vie (inaudible) quelque chose de l'ordre de l'expérience commune qui à la fois va me servir de comparaison pour donner à comprendre ce qu'il y a à comprendre dans l'Evangile parce que si je dis directement les choses on n'écoute pas et on ne comprend pas mais avec une comparaison bien sentie on comprend et ensuite du coup ce que dit l'Evangile va éclairer la situation de vie mise en ouvre. Voilà. D'où en effet comme tu disais quand ça quand ça réussit un croisement. 
A 15 Oui parce qu'il y a un croisement tout à fait... Alors comment justement ce croisement tu l'as conçu tu l'as conçu dans ses grandes lignes...

M 16 Dans son principe. Voilà ce que j'ai dit dans son principe je l'ai conçu et après ça en gros je dis ben je vais y aller je vais aller de a à $\mathbf{z}$ comme ça

A 17 Tu as des points quoi en fait.

M 18 J'ai le chemin. J'ai le chemin. (geste de la main en zigzags)

A 19 Oui. Et après (inaudible)

M 20 (inaudible)

A 21 en parlant

M 22 en parlant

A 23 Non parce que c'est un Evangile qui était... qui est loin d'être commode.

M 24 Oui c'est vrai oui bien sûr c'est particulièrement coton.

A 25 (inaudible)

Ch 26 Et vous aviez un chemin qui était un peu comme ça là quand vous le dessiniez à l'instant. (en reproduisant le geste de zigzag de $M$ )

M 27 Ah oui je... Oui. Parce que il n'est pas du tout toujours le même. Il y a des choses pour aller jusque là il va falloir que je dise certaines choses et en fait ce n'est pas forcément un ordre simple c'est voilà la situation de vie 12 voilà l'Evangile ce que ce qu'on entend de manière à comprendre 3 la conclusion voilà l'éclairage pour nous. Mais en réalité le le chemin n'est pas...

Ch 28 Non c'est comme ça

M 29 Il y a recouvrements il y a des appels il y a des échos

A 30 Moi ce que je dirais à propos de ça en (inaudible) c'est que le cheminement n'est pas un cheminement strictement logique tout au moins didactique mais c'est plus des... comme des zones de couleur qui sont placées dans les mêmes tonalités qui sont placées l'une à côté de l'autre et qui font effectivement qu'il y a le cheminement c'est... Le cheminement n'est pas que c'est pas un cheminement démonstratif au sens d'un d'un d'une dissert quoi. Il y a quelque chose de de je trouve plus... qui se rapproche du récit par certains côtés tu mets en scène et tes et tu rapproches euh et de là naît enfin c'est comme ça que j'ai suivi et que je le comprends d'abord l'envie de penser pour savoir où tu vas arriver et puis cette force de la parole qui est une force... qui tient pas simplement qu'à la consistance mais qui tient aussi à cette méthode. C'est comme ça que je comprends le zigzag entre guillemets qui qui donne ta position. Mais j'en sais rien si ça permet je ne sais pas si c'est juste quoi.

M 31 Mais j'ai l'impression de reconnaître oui

A 32 Des plans des séquences

M 33 Oui oui oui.

A 34 Mais le rapprochement fait jaillir comme par surcroît un sens que tu ne dis pas forcément explicitement même si tu l'as élucidé à la fin. Je trouve que c'est du... grand art

\subsection{2.- Analyse détaillée des deux mouvements de double migration}

Premier mouvement : à partir de 11, «dis moi dis moi ça m'intéresse », migration du «travail avec la traduction liturgique et la version originale» d'instrument de l'activité pratique à instrument de l'argumentation en ACC puis à objet de l'activité d'analyse dans l'ACC.

Le travail de lecture comparée qui fait appel à différentes traductions de la Bible peut être considéré comme un instrument générique pour les prêtres. Il n'est pas l'apanage de quelques individus mais un outil de travail ordinaire pour tous les prêtres amenés à prêcher régulièrement pour leurs paroissiens. A proprement parler, cet instrument s'appuie sur un système d'artefacts : la Bible en version originale, sa traduction liturgique, d'autres traductions en français ou dans d'autres langues, des règles d'interprétation des textes, des notes exégétiques, etc. 


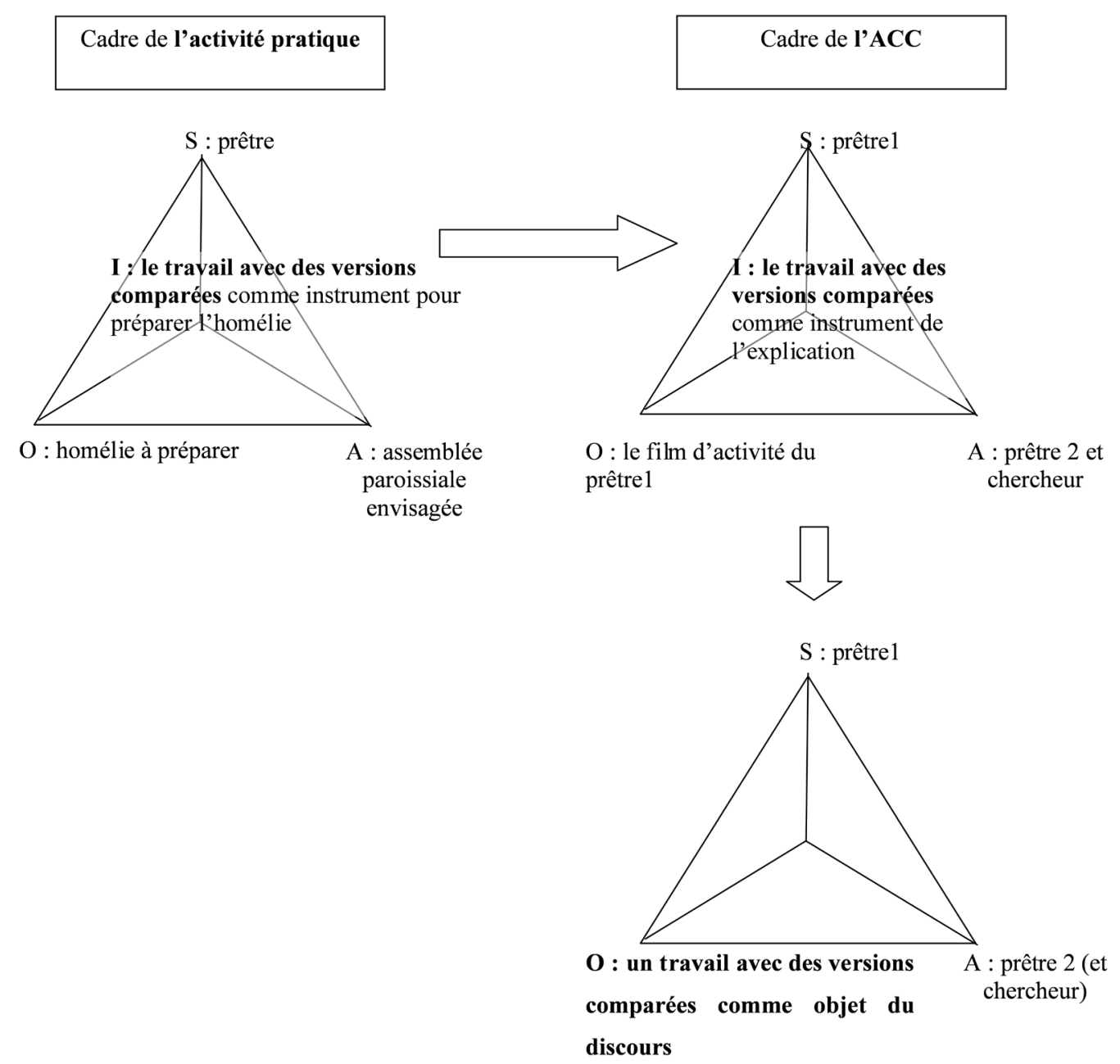

Figure 4 : de l'instrument de métier à l'objet du discours : le cas des versions comparées

Figure 4: From work tool to object of discourse : the case of compared translations

Nous retenons le moment dans le cadre de l'autoconfrontation croisée où cet instrument générique ordinairement mobilisé dans l'activité pratique (plus précisément ici lors de la préparation de l'homélie) apparaît dans un épisode conversationnel, lors de l'activité d'analyse de l'homélie, en position d'instrument de l'argumentation chez l'un des prêtres. M, tentant d'expliquer sa façon de procéder à son confrère, introduit en 2, 4 et 10 le rôle central des textes bibliques et de ce principe de lecture comparée. Mais cette explication reste abstraite. C'est le passage d'une description générale de sa façon de faire (qui mentionne au passage la lecture comparée des textes bibliques) à une illustration concrète de ce que cette façon de faire apporte qui initie le double mouvement qui retient ici notre attention. L'apparition dans le discours de $\mathrm{M}$ de ce qu'on pourrait qualifier avec J.P. Bronckart et $\mathrm{E}$. Bulea (Bronckart \& Bulea, 2006 ; Bulea, 2007) de figure d'action-occurrence introduite par la rupture « par exemple dimanche prochain... » suscite la curiosité de son interlocuteur : A demande des précisions à ce sujet. A l'instigation de son confrère (en 11), M est poussé à reprendre et développer son argumentaire autour de son usage des divergences entre la version originale et la traduction liturgique (en 12). De ce moment, ce qui était un instrument destiné à étayer une explication (l'exemple de dimanche prochain) devient le cœur du discours, son objet, vers lequel sont tournées les activités de $\mathrm{M}$ et d'A jusqu'au nouveau changement de ton du milieu de l'intervention 14 : «D'ailleurs justement souvent... » qui signe le passage, toujours selon la typologie de J.P. Bronckart et E. Bulea, à une figure d'action-définition, et la fin du processus. Nous retenons pour notre part cette migration 
fonctionnelle, initiée par le questionnement du pair, où l'apport de la version originale à la traduction liturgique devient l'objet de la discussion et temporairement, l'objet même de l'analyse conjointe. La façon d'utiliser les variations de traduction devient l'objet même sur lequel se focalise l'activité d'analyse des deux prêtres.

Deuxième mouvement : à partir de 26, intervention du chercheur " et vous aviez le chemin », migration du « chemin en zigzag» d'instrument à objet de l'activité d'analyse.

C'est ici une notion de métier, «le chemin », qui connaît une double migration :

- Elle passe d'abord, avec le changement de cadre de l'activité pratique à l'activité d'analyse en autoconfrontation croisée, du statut d'instrument générique dans l'activité pratique à celui de ressource argumentative dans l'activité d'analyse ;

- Puis, à l'initiative cette fois-ci du chercheur dans une reprise que nous détaillons ci-dessous, du statut de ressource argumentative à celui d'objet même de l'analyse.

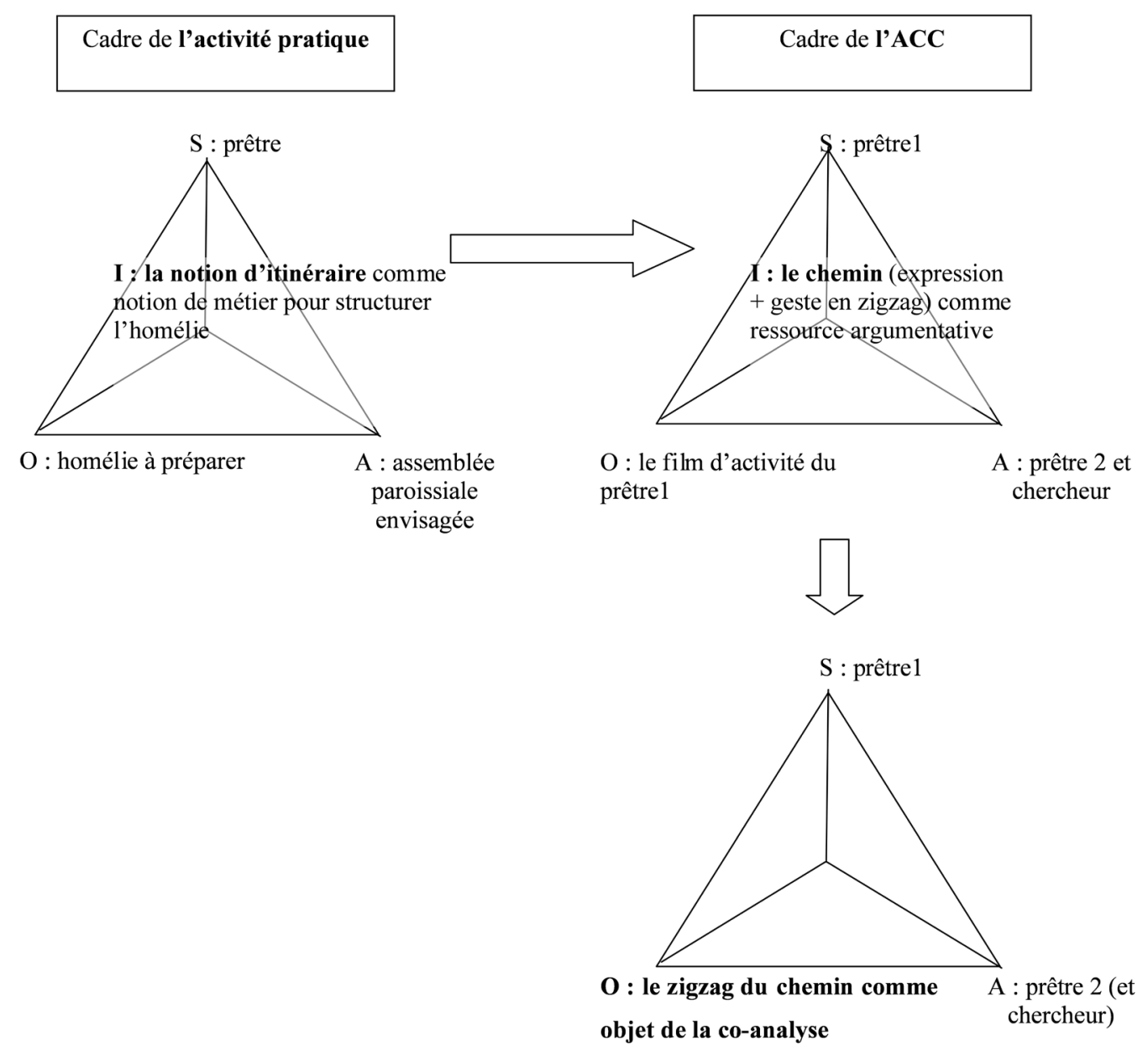

Figure 5 : de l'instrument de métier à objet du discours : le cas du «chemin »

Figure 5: From work tool to object of discourse : the case of «the path»

Nous avons vu que la séquence S2 s'ouvrait sur un questionnement d'A, qui relevait à partir du film de son confrère ce qu'il qualifiait de « croisement» remarquable et dont il cherchait à comprendre la genèse. C'est la reprise en 15 de ce questionnement qui amène $M$ à expliquer qu'il a « le chemin » (la progression entre 15 et 18 mène à l'apparition de cette expression plus juste que celle du «plan »). L'apparition de ce terme de métier (le chemin) s'accompagne d'un geste illustratif de la main dessinant un zigzag dans l'espace de la pièce où se déroule l'autoconfrontation croisée. La notion de 
métier (le chemin autour duquel se construit l'homélie) est d'abord mobilisée comme ressource argumentative pour répondre au questionnement insistant du pair.

Toutefois, le caractère même de cette expression (c'est-à-dire son statut de notion de métier partagée par les acteurs initiés à l'activité) interdit quasiment la poursuite du questionnement et de l'échange, puisqu'en tant que professionnel, A est censé savoir de quoi $\mathrm{M}$ parle. D'ailleurs, après un bref échange, la conversation s'épuise comme le montre l'apparition d'une forme d'agrément générique (en 24-25) : la reconnaissance par A de la complexité du texte immédiatement validée par M. On trouve à ce moment de l'autoconfrontation croisée une forme typique de l'échange dans ce cadre dialogique : l'accord mutuel qui signe moins la satisfaction de l'échange sur le fond qu'il ne confirme le statut social des sujets, validant mutuellement qu'ils font le même métier, partagent les mêmes soucis, techniques, appréciations et valeurs.

La reprise par le chercheur (en 26) du terme apparu dans la bouche de M (en 18), c'est-à-dire de l'expression "j'ai le chemin », et du geste même de zigzag qui l'accompagnait, fait entrer ce chemin en tant que tel dans l'espace de l'analyse : d'élément de réponse à une question du confrère, il devient l'objet de l'explicitation et de l'investigation. C'est le changement de destinataire qui transforme le statut de l'expression et lui donne dans la conversation une chance de vivre une « deuxième vie ». On peut penser que la réussite de cette transformation est toutefois rendue possible par l'implication d'A dans l'analyse, chez qui le questionnement sur la genèse du croisement imaginé par son confrère n'est sans doute pas éteint. La poursuite de l'investigation est ainsi rendue possible par le commentaire qu'A apporte à la description par $\mathrm{M}$ de la nature de ce « chemin » dans son homélie. Cette focalisation sur une notion de métier qui fonctionne habituellement comme un instrument générique dans l'activité pratique permet un approfondissement potentiel de l'analyse des deux prêtres et du chercheur.

\section{3.- Discussion}

Notre attention a été retenue par certaines similitudes que présentent les réalisations langagières analysées en dépit de leur diversité. En effet, entre ces séquences, beaucoup de différences existent : la nature de l'activité commentée, la phase méthodique du commentaire (autoconfrontation simple ou croisée), les destinataires... Pourtant, malgré ces différences initiales, une analogie est remarquable : celle d'un processus de double migration qui constitue le préalable à un autre regard du professionnel sur son activité. Un élément de l'activité pratique est mobilisé d'abord dans le cadre dialogique de l'autoconfrontation comme ressource argumentative pour répondre à une interrogation du chercheur (dans le cadre de l'autoconfrontation simple ou croisée) ou du pair (dans le cadre de l'autoconfrontation croisée) initiée par le visionnage du film de l'activité ; puis une intervention du chercheur ou du pair transforme cette ressource argumentative en objet de discours autonome, permettant que la conversation se déploie avec temporairement cet objet comme objet principal d'analyse. Cette intervention prend souvent la forme d'une reprise ${ }^{11} \mathrm{~d}$ 'un « mot-clef» :

$$
\begin{aligned}
& \text { C } 11 \text { vous dites que ça peut les guider? } \\
& \text { C } 35 \text { vous disiez c'est une forme de reconnaissance? } \\
& \text { C } 26 \text { et vous aviez un chemin (accompagné de la reprise du geste de zig zag) }
\end{aligned}
$$

Pourtant, la reprise d'une séquence du discours n'est pas suffisante pour provoquer les réalisations langagières que nous observons ici (en particulier, la construction d'un objet de discours et d'analyse). On trouve dans les mêmes matériaux de nombreux exemples de reprise qui ne donnent pas lieu aux mêmes développements.

Par exemple, dans l'intervention sur la VAE, une collègue de JF commente la même tâche, l'entretien de jury avec une candidate, en autoconfrontation simple avec le même chercheur. Elle a à ce moment

\footnotetext{
11. Nous choisissons d'utiliser le terme de « reprise » proposé notamment par Vion (2006), de préférence par exemple à « reformulation »: sa caractéristique générique permet de considérer une relation au déjà-dit, dont les rapports et les formes de similitude peuvent varier.
} 
sous les yeux les images d'elle-même en train d'écouter la candidate animatrice qui commente l'une des activités qu'elle a réalisée avec de jeunes enfants.

J 43 oui alors là mon souci ... dans son dossier, elle cite des démarches pédagogiques... mais là je la vois pas... dans cette activité là on la voit bien préparer sa salle elle explique comment elle prépare sa salle, comment elle prend en compte l'espace avec les enfants mais elle n'écrit pas comment elle s'adapte à un public ....avec des tranches d'âge....où ça bouge beaucoup quand même ... et donc mon souci c'était concrètement comment elle fait

Ch 44 vous dites on la voit pas?

J 45 non on la voit pas, enfin, on la voit pas... dans sa description on ne la voit pas animer cet atelier précisément... donc ben... je l'attends....

Ou encore, un peu plus loin, commentant le fait qu'elle pose une question à la candidate alors que son collègue vient juste d'en poser une :

J 114 là je reprends la main... d'ailleurs à un moment je fais un peu ça (fait un geste)

$\mathrm{Ch} 115$ vous dites je reprends la main ?

J 116 oui, je reprends la main parce que ben j'avais d'autres questions d'abord... et puis je me suis dit ... il faut pas que ça... que ça s'enchaîne sur des jugements de valeur et là on était parti sur des jugements de valeur dans cette question un peu donc j'ai repris la main pour éviter ça

La reprise ne provoque pas ici les effets de construction d'un objet de discours que nous avons $a$ contrario identifiés dans les séquences présentées précédemment dans cet article. Elle ne provoque pas non plus les effets d'interrogation que nous pouvons y observer. En effet, les dialogues qui font suite aux doubles migrations précédemment analysées se signalent par une autre analogie remarquable : ce qui jusque là relevait de l'évidence générale perd sa caractéristique d'invisibilité. On le sait, plus les professionnels ont de l'expérience, plus celle-ci disparaît à leurs yeux : "incorporée » (Leplat, 1995), elle échappe à ceux qui la portent. ${ }^{12}$. Or trois ${ }^{13}$ des situations analysées ici montrent précisément, juste après le deuxième mouvement de migration c'est-à-dire la reprise par le chercheur, une suspension du flux discursif du professionnel engagé dans l'analyse. Cette suspension se manifeste par des interruptions de quelques secondes. A la suite de ces interruptions, de nouvelles manières de réaliser langagièrement l'activité pratique émergent, en en soulignant notamment les différences, les variations. Elles s'accompagnent également parfois de manifestations émotionnelles, sourires ou rires.

Interruptions :

27. $M$ : Ah oui je...(interruption) Oui.

$12 \mathrm{JF}$ : alors je sais pas si c'est guider mais enfin c'est nous c'est...(interruption) bon...

(interruption) je sais pas

$36 \mathrm{JF}$ : alors reconnaissance... je sais pas si c'est le mot mais enfin en tout cas (interruption)

Réalisation de différences :

27 M : Parce que il n'est pas du tout toujours le même

$12 \mathrm{JF}$ on n'est pas en formation ou en certification

Rires :

$12 \mathrm{JF}$ : alors je sais pas si c'est guider mais enfin c'est nous c'est. Bon. je sais pas j'ai pas trop analysé... en tout cas, on a l'habitude de le faire (rire)

Et plus loin dans le même tour de parole :

ben qui on est aussi ça fait partie des... de ce qu'on peut enseigner aussi (rire)

12. Elle échappe alors tout autant à un observateur qui souhaite la voir la comprendre, l'expliquer, l'analyser.

13. Nous ne commentons pas ici la quatrième situation, mais il est à noter que dans cette situation la reprise est effectuée par le pair et non par le chercheur. 
$36 \mathrm{JF}$ : alors reconnaissance...je sais pas si c'est le mot mais enfin en tout cas dire que... son dossier a été... lu avec intérêt (rire)

Et plus loin dans le même tour de parole :

c'est aussi pour...comment je vais dire...éviter... oui que...que... la subjectivité quoi je veux pas dire qu'on est objectif (rire) mais on essaie d'avoir d'aller vers le plus d'objectivité possible

Ces suspensions du flux discursif, qui signent l'absence de mots pour dire, témoignent de l'impossible mobilisation d'un « déjà dit » (Bournel-Bosson, 2005). Elles ouvrent alors potentiellement la voie de nouvelles réalisations. Le commentaire engage ensuite une densification des manières possibles ou effectives de conduire l'activité pratique, avec ses conflits potentiels. Autrement dit, la dénaturalisation des mots dans le périmètre de l'autoconfrontation, qui perdent l'évidence de leur sens habituel, peut conduire à d'autres manières de réaliser ce qui est fait, au-delà de son évidence et son opacité.

La réussite de ce double processus de migration pour soutenir l'activité de pensée et d'analyse des sujets, qui se manifeste par des réalisations langagières spécifiques, est donc loin d'être automatique. Un retour sur les modalités précises du processus dans les séquences analysées permettra de dégager un élément essentiel de cette réussite :

\section{1 - Migration d'un instrument de l'activité pratique dans l'activité langagière}

Un instrument générique de l'activité pratique (la lecture comparée à partir de la traduction liturgique, la notion d'itinéraire, la notion de guidage ou celle de reconnaissance, si elles n'ont pas le même statut, ont en commun de cristalliser en elles une histoire du métier) devient une ressource pour l'argumentation en autoconfrontation (simple ou croisée). Il y a une migration interactivités (de l'activité pratique à l'activité conduite en autoconfrontation croisée) à fonction constante, celle d'instrument.

$$
\begin{aligned}
& \text { JF } 10 \text { çà peut aussi les guider } \\
& \text { JF } 34 \text { c'est une forme de reconnaissance } \\
& \text { M } 10 \text { En fait la traduction liturgique } \\
& \text { M } 18 \text { J'ai le chemin (+ geste de la main en zig zag) }
\end{aligned}
$$

\section{2 - Reprise d'un « mot-clef» (segment du discours, expression ou mot, parfois accompagné d'un geste) par le chercheur, ou questionnement direct par le pair}

La technique de reprise par le chercheur d'un segment du discours ouvre à une transformation du statut de ce dernier. De ressource argumentative, il peut devenir objet d'une activité conjointe d'analyse. De la même façon, le questionnement par le pair arrête la progression du discours et transforme le statut de ce qui vient d'être dit.

$$
\begin{aligned}
& \text { C } 11 \text { vous dites que ça peut les guider? } \\
& \text { C } 35 \text { vous disiez c'est une forme de reconnaissance? } \\
& \text { AL } 11 \text { dis moi ça m'intéresse } \\
& \text { C } 26 \text { et vous aviez un chemin (+ geste en zig zag) }
\end{aligned}
$$

\section{3 - Migration fonctionnelle d'instrument à objet en autoconfrontation croisée}

L'instrument de l'activité argumentative devient l'objet partagé d'une interrogation. Il change ainsi de fonction ou plutôt en conquiert une nouvelle, en migrant d'une position instrumentale à celle d'objet temporaire de l'analyse. L'échange en autoconfrontation simple ou croisée permet ainsi la construction d'un objet commun qui n'était pas donné a priori par le film de l'activité. Cet objet a certaines propriétés dont la caractérisation précise nécessite un travail ultérieur. En première approximation, on peut toutefois soutenir que cet objet est : (a) un objet «d'analyse » au sens étymo$\operatorname{logique}^{14}$, c'est-à-dire un objet « délié », porteur de multiples points de vue, (b) « en commun » aux

14. du grec $\alpha \dot{v} \alpha \lambda v \omega \omega$, analuein signifiant « délier ». 
interlocuteurs, et (c) en lien avec un concept du métier, cristallisant un conflit générique.

\section{Conclusion}

Notre question première était celle des conditions dans lesquelles un commentaire du travail, dans un certain cadre dialogique, permettait aux professionnels de mettre en place une analyse de leur activité ouvrant à des développements potentiels de cette dernière. Le processus mis ici au jour permet d'avancer en ce sens (avec la limite fixée d'emblée à notre projet : nous ne nous intéressons pas ici aux effets potentiels de cette analyse sur l'activité pratique, c'est-à-dire aux rapports entre transformations de l'activité d'analyse et transformations de l'activité de travail). Le cadre dialogique ne suffit pas en effet à garantir l'efficacité de la construction discursive. Ce n'est pas seulement la focalisation sur un objet de discours commun qui lui donne un potentiel de développement de l'activité de pensée des sujets. On peut parler ensemble du même objet sans le déplacer le moins du monde ni se déplacer soi-même. Or, la construction du nouveau suppose un tel déplacement. Dans les séquences analysées, nous avons relevé deux points intéressants relatifs à la nature même de l'objet qui permettent ces développements :

- l'objet du discours, avant d'accéder à cette fonction, a une vie propre dans le métier analysé : celle de notion ou concept de métier, celle d'instrument de travail pour le sujet, ici d'instrument psychologique ;

- Il émerge comme objet de discours et d'analyse dans l'interaction. Dans les cas analysés, c'est la médiation par un tiers qui permet cette émergence. Il apparaît alors là où il est potentiellement atteignable par les sujets et par le chercheur, comme une ressource argumentative. Puis sa reprise transforme son statut, d'instrument de l'argumentation à objet d'interrogation et d'analyse, permettant l'ouverture d'un espace de pensée dans le dispositif dialogique. Dans la limite des matériaux ici présentés, les manifestations de cet espace ne se réalisent pas de manière formellement identique au plan langagier, selon que la reprise est portée par le chercheur ou par le pair : il conviendra d'instruire plus précisément cette différence recoupant en partie le caractère simple ou croisé des phases d'autoconfrontation.

ANNEXE : CONVENTIONS DE RETRANSCRIPTION

A, JF, M ... : professionnel

$\mathrm{Ch}:$ chercheur

... syllabe allongée, silence

? marque interrogative

en gras : les passages source des analyses

(en italique) notes de retranscription, par exemple, pour signaler des mots inaudibles ou des manifestations non langagières

\section{RÉFÉRENCES}

Bakhtine, M. (1984). Esthétique de la création verbale. Paris: Gallimard.

Béguin, P., \& Cerf, M. (2004). Formes et enjeux de l'analyse de l'activité pour la conception des systèmes de travail. Activités, 1(1), 54-71. http://www.activites.org/v1n1/beguin.pdf

Bournel-Bosson, M. (2005). Les organisateurs du mouvement dialogique : autoconfrontations croisées et activité des conseillers en bilans de compétence. Thèse de doctorat en psychologie. Paris: CNAM.

Bronckart, J.P., \& Bulea, E. (2006). La dynamique de l'agir dans la dynamique langagière. In J.M. Barbier \& M. Durand (Eds.), Sujet, activité, environnement : approches transverses (pp. 105-134). Paris: PUF. 
Bulea, E. (2007). Le rôle de l'activité langagière dans l'analyse des pratiques à visée formative. Thèse de doctorat de psychologie. Genève: Université de Genève.

Clot, Y. (1999). La fonction psychologique du travail. Paris: PUF.

Clot, Y. (2004). Le travail entre fonctionnement et développement. Bulletin de Psychologie, 57(1), 5-12.

Clot, Y. (2005). L'autoconfrontation croisée en analyse du travail : l'apport de la théorie bakhtinienne du dialogue. In L. Filliettaz \& J.P. Bronckart (Eds.), L'analyse des actions et des discours en situation de travail (pp. 37-55). Louvain-La-Neuve: Peeters.

Clot, Y. (2008). Travail et pouvoir d'agir. Paris: PUF.

Clot, Y., Faïta D., Fernandez G., \& Scheller, L. (2001). Les entretiens en autoconfrontation croisée : une méthode en clinique de l'activité. Education permanente, 146, 17-25.

Engeström, Y. (1987). Learning by expanding: An activity-theoretical approach to developmental research. Helsinki: Orienta-Konsultit.

Engeström, Y. (2000). Activity theory as a framework for analyzing and redesigning work. Ergonomics, 43 (7), 960-974

Engeström, Y., Virkkunen, J., Helle, M., Pihlaja, J., \& Poikela, R. (1996). The Change laboratory as a tool for transforming work. Lifelong Learning in Europe, 1(2), 10-17.

Filliettaz, L., \& J.P. Bronckart (2005). L'analyse des actions et des discours en situation de travail. Concepts, méthodes et applications. Louvain-La-Neuve : Peeters.

Kloetzer, L. (2008). Analyse de l'homélie de la messe dominicale : langage et conflits de métier dans l'activité des prêtres. La part de Dieu, la part de l'homme. Thèse de doctorat en psychologie. Paris: CNAM

Kostulski, K. (2005). Activité conversationnelle et activité d'analyse : l'interlocution en situation de coanalyse du travail. In L. Filliettaz \& J.P. Bronckart (Eds.), L'analyse des actions et des discours en situation de travail (pp. 57-74). Louvain-La-Neuve: Peeters.

Kostulski, K., \& Clot, Y. (2007). Interaction et migration fonctionnelle. Un développement en autoconfrontation croisée. Psychologie de l'interaction, 23-24, 73-108.

Leplat, J. (1995). A propos des compétences incorporées. Education Permanente, 123, 101-114.

Pastré, P. (2005). La conception de situations didactiques à la lumière de la théorie de la conceptualisation dans l'action. In P. Rabardel \& P. Pastré (Eds.), Modèles du sujet pour la conception. Dialectiques activité développement (pp. 73-107). Toulouse: Octarès.

Perret-Clermont, A.N. (2001). Psychologie sociale de la construction de l'espace de pensée. In J. J. Ducret (Ed.), Actes du colloque. Constructivisme: usages et perspectives en éducation (pp. 65-82). Genève: Département de l'Instruction Publique, Service de la recherche en éducation.

Vion, R. (2006). Reprise et modes d'implication énonciative. La linguistique, 42(2), 11-25.

Vygotski, L. S. (1978). Mind in society. Harvard: Harvard University Press

Vygotski, L.S. (1934/1997). Pensée et langage. Paris: La Dispute.

Vygotski, L.S. (1927/1999). La signification historique de la crise en psychologie. Neuchâtel: Delachaux et Niestlé.

Wallon, H. (1983). Les origines du caractère chez l'enfant. Paris: PUF.

Wisner, A. (1997). Aspects psychologiques de l'anthropotechnologie. Le Travail humain, 60(3), 229-254. 


\section{RÉSUMÉ}

De plus en plus de méthodologies d'analyse du travail revendiquent une approche développementale, c'est-à-dire visent la transformation des situations et/ou des individus. Un certain nombre d'entre elles postulent que c'est en permettant aux professionnels d'engager eux-mêmes une analyse de leur propre activité de travail qu' on y parvient (voir par exemple Clot, 2008 ; Engeström, 1987, 2000 ; Béguin \& Cerf, 2004 ; Pastré, 2005). Toutefois, l'expérience montre que même au sein de dispositifs soigneusement construits pour favoriser le développement de leur activité par les sujets, les moments de développement véritable de l'activité d'analyse, sans parler même du développement de l'activité pratique, qui reste en général difficile à attester dans l'espace-temps du dispositif de recherche, sont des « perles rares ». Nous nous penchons dans cet article sur quelques unes de ces perles, isolées au sein de deux interventions conduites en clinique de l'activité avec la méthodologie des autoconfrontations croisées (Clot, 1999, 2005, 2008 ; Clot et al., 2001), l'une avec des experts de la VAE et l'autre avec des prêtres catholiques, afin d'extraire de leur comparaison une meilleure compréhension des conditions du développement de l'analyse. Nous nous focaliserons dans cet article sur un des mécanismes, récurrent dans les matériaux analysés, qui contribue à la construction de cet « espace de pensée » (Perret-Clermont, 2001) où l'on parvient à produire du nouveau.

\section{MoTs CLÉS}

analyse du travail, activité, dispositifs dialogiques, autoconfrontation croisée, développement, migration fonctionnelle

\section{RÉFÉRENCEMENT}

Kloetzer, L. \& Henry, M. (2010). Quand les instruments de métier deviennent objets de discours : une condition de l'analyse du travail en autoconfrontation croisée ? Activités, 7(2), 44-62, http://www. activites.org/v7n2/v7n2.pdf.

Article soumis le 7 octobre 2009, accepté pour publication le 20 avril 2010. 